\title{
A yield criterion for anisotropic and pressure dependent solids such as oriented polymers
}

\author{
ROBERT M. CADDELL, RAM S. RAGHAVA, ANTHONY G. ATKINS \\ Department of Mechanical Engineering, University of Michigan, Ann Arbor, Michigan, USA
}

The anisotropic yield criterion first posed by Hill has been modified to account for differences in tensile and compressive yield strengths in a given direction; additionally, the influence of hydrostatic pressure on yielding is also considered. Predictions using this new criterion are compared with published experimental results involving oriented polymers and excellent agreement is found. It is suggested that this criterion is more correct on fundamental grounds than those put forth in earlier publications.

\section{Introduction}

The macroscopic yielding behaviour of isotropic polymers has been studied during recent years by various authors [1-5] and from those publications have come different phenomenological yield criteria. A detailed comparison of those studies was made by Raghava [6] and a subsequent publication [7] supports the stand that the criterion proposed in [5] predicts more realistically the yielding behaviour of polymers under increasing hydrostatic pressure than do others [1-4]. There is, however, unanimous agreement among all of the aforementioned investigators that the yield behaviour of polymers is influenced by the magnitude of the mean normal stress (i.e. the behaviour is "pressure sensitive").

Fewer studies have been carried out in regard to the yield behaviour of anisotropic polymers. The work of Brown et al [8], Rider and Hargreaves [9], Rawson and Rider [10], and, most recently, Shinozaki and Groves [11] are typical. Hill's "anisotropic" yield criterion [12] has been used [8-10] as a basis from which to explain the experimental results but a modifying parameter (denoted as $\sigma_{i}$ or $a_{x x}$ ), had to be included to produce a reasonable correlation. This parameter was denoted as a "Bauschinger" term and was considered to account for the effect of "internal" stresses developed during the orientation process that produced an anisotropic material. Rawson and Rider [10] noted that this term was not an arbitrary parameter but was related to the yield strengths in tension and compression for a particular direction in the oriented sheet used by them.

Hill's basic criterion [12] does not include linear terms since no Bauschinger effect is assumed thus, in any angular orientation, the tensile and compressive yield strengths are equal in magnitude. Another implicit condition relates to the assumption that there is no change in plastic volume during deformation. Neither of these assumptions is valid for polymeric solids [1, 8-11], so one should not expect the Hill criterion to find direct application with such solids unless modifications are made. Now the use of an "internal" stress term [8-10] may satisfy one of these assumptions but the improved correlation that results between experiment and this modified theory is perhaps fortuitous at best and a fudge factor at least. This was recognized apparently by Rawson and Rider [10] who modified an earlier suggestion [4] but they were not fully successful.

What is crucial here is that a yield criterion for anisotropic polymers must account directly for differences in tensile and compressive yield strengths and accommodate changes in plastic volume if it is to satisfy observed evidence. The criteria proposed to date [8-11] do not. This paper uses as a starting point the pressuredependent criterion proposed for isotropic polymers [5-7] and by adapting this to a Hill type of approach, leads to a proposed criterion that seems more direct and more fundamentally 
sound than any put forth to date. Agreement with results already published in the literature $[10,11]$ is excellent.

\section{Development of the suggested anisotropic yield criterion}

To most readily explain the rationale behind the proposed end result, first consider the yield criterion that really forms the starting point for this whole discussion. This is the criterion most widely attributed to von Mises and applies to isotropic solids that exhibit no Bauschinger effect and whose yield behaviour is unaffected by the magnitude of the mean normal stress (i.e. the superposition of a hydrostatic stress does not influence yielding). One form is expressed as, $\left(\sigma_{x}-\sigma_{y}\right)^{2}+\left(\sigma_{y}-\sigma_{z}\right)^{2}+\left(\sigma_{z}-\sigma_{x}\right)^{2}$

$$
+6\left(\tau_{x y}{ }^{2}+\tau_{y z}{ }^{2}+\tau_{z x}{ }^{2}\right)=2 Y^{2} \text {. }
$$

In Equation 1, the yield strength, $Y$, is assumed equal in tension and compression. Raghava et al [5-7] have proposed a criterion for isotropic but pressure sensitive solids (such as polymers) which is a modification of Equation 1; it is,

$$
\begin{aligned}
\left(\sigma_{x}-\sigma_{y}\right)^{2} & +\left(\sigma_{y}-\sigma_{z}\right)^{2}+\left(\sigma_{z}-\sigma_{x}\right)^{2} \\
& +6\left(\tau_{x y}{ }^{2}+\tau_{y z}{ }^{2}+\tau_{\sigma_{x}}{ }^{2}\right) \\
& +2(C-T)\left(\sigma_{x}+\sigma_{y}+\sigma_{z}\right)=2 C T
\end{aligned}
$$

where $C$ and $T$ are the absolute values of compressive and tensile yield strength measured at atmospheric pressure. The pressure influence is accounted for by the quantity $\left(\sigma_{x}+\sigma_{y}+\sigma_{z}\right)$ and it may be noted that if $C$ and $T$ are equal, Equation 2 reduces to Equation 1.

Hill's approach to the problem of plastic anisotropy was to modify Equation 1 in the following form,

$$
\begin{aligned}
H\left(\sigma_{x}-\sigma_{y}\right)^{2} & +F\left(\sigma_{y}-\sigma_{z}\right)^{2}+G\left(\sigma_{z}-\sigma_{x}\right)^{2} \\
& +2 N \tau_{x y}{ }^{2}+2 L \tau_{y z}{ }^{2}+2 M \tau_{z x}{ }^{2}
\end{aligned}=1 .
$$

The parameters $F, G, H, L, M$ and $N$ characterize the state of anisotropy. Here, $x, y$ and $z$ denote the principal axes of anisotropy as well as the reference axes. Thus, if a polymeric sheet is oriented by stretching, for example, $x$ may be viewed as the axis along which stretching occurred, $y$ is at right angles to $x$ and in the plane of the sheet, and $z$ is normal to the plane of the sheet.

Now Equation 3 is based upon the same assumptions attributed to Equation 1 except for the "anisotropic" parameters. As this effect becomes small, the parameters themselves relate in such a way that Equation 3 reduces to Equation 1. What we propose is to revise Equation 3 to produce a pressure dependent, anisotropic yield criterion along the lines that Equation 2 follows from Equation 1. Thus, the proposed form is,

$$
\begin{gathered}
H\left(\sigma_{x}-\sigma_{y}\right)^{2}+F\left(\sigma_{y}-\sigma_{z}\right)^{2}+G\left(\sigma_{z}-\sigma_{x}\right)^{2} \\
2 N \tau_{x y}{ }^{2}+2 L \tau_{y z}{ }^{2}+2 M \tau_{z x}{ }^{2} \\
+K_{x} \sigma_{x}+K_{y} \sigma_{y}+K_{z} \sigma_{z}=1
\end{gathered}
$$

where the various parameters depend upon the absolute values of tensile and compressive yield strengths in the three reference directions. These are, $C_{x}, C_{y}$ and $C_{z}$ (compression) and $T_{x}, T_{y}$ and $T_{z}$ (tension). The parameters are,

$$
\begin{array}{r}
H+G=\frac{1}{C_{x} T_{x}}, F+H=\frac{1}{C_{y} T_{y}}, \\
G+F=\frac{1}{C_{z} T_{z}}
\end{array}
$$

and

$$
\begin{array}{r}
K_{x}=\frac{C_{x}-T_{x}}{C_{x} T_{x}}, K_{y}=\frac{C_{y}-T_{y}}{C_{y} T_{y}}, \\
K_{z}=\frac{C_{z}-T_{z}}{C_{z} T_{z}} .
\end{array}
$$

Certain special cases may be noted using Equation 4 and the relations given in Equations 5 and 6 . These are:

1. if $C_{x}=C_{y}=C_{z}=T_{x}=T_{y}=T_{z}$, Equation 4 reduces to Equation 1,

2. if $C_{x}=T_{x}, C_{y}=T_{y}, C_{z}=T_{z}$ but $T_{x} \neq$ $T_{y} \neq T_{z}$, Equation 4 reduces to Equation 3, 3. if $C_{x}=C_{y}=C_{z}$ and $T_{x}=T_{y}=T_{z}$ but $C_{x} \neq T_{x}$, Equation 4 reduces to Equation 2. Thus Equation 4 can be viewed as a more universal criterion from which other, more widely used criteria may be developed because of particular relationships of pertinent property values.

Consider a stretched or rolled sheet of polymer from which the compressive and tensile properties are to be investigated for various orientations with respect to the oriented direction (taking $x$, $y$, and $z$ in the same context as described under Equation 3). Standard strip tensile specimens, cut at an angle $\theta$ to the " $x$ direction" would be subjected to a stress, $\sigma$, at which yielding occurs. (Note a parallel description applies to compression tests.) For such tests,

$$
\sigma_{z}=\tau_{y z}=\tau_{z x}=0
$$

and such that at the onset of yielding in the " $\theta$ direction",

$$
\begin{aligned}
& \sigma_{x}=\sigma \cos ^{2} \theta \\
& \sigma_{y}=\sigma \sin ^{2} \theta \\
& \tau_{x y}=-\sigma \sin \theta \cos \theta .
\end{aligned}
$$


Substitution of Equations 7 and 8 into Hill's criterion (Equation 3) leads to,

$$
\begin{aligned}
\sigma^{2}\left[(G+H) \cos ^{4} \theta\right. & +(H+F) \sin ^{4} \theta \\
& \left.+2(N-H) \sin ^{2} \theta \cos ^{2} \theta\right]=1 .
\end{aligned}
$$

Now it is apparent that once the parameters $(G+H),(H+F)$ and $(N-H)$ are evaluated, Equation 9 predicts two roots of equal absolute value for a given value of $\theta$. With metallic solids this has often been verified*. For some of the reasons mentioned earlier, the situation with polymers is different so Equation 9 should not be expected to describe the yield behaviour of anisotropic polymers in an acceptable manner. Shinozaki and Groves [11] noted this quandary when applying Equation 9 while others [8-10] altered Equation 9 with the "internal" stress term mentioned earlier.

Consider the proposed criterion; by substituting Equations 7 and 8 into Equation 4 one finds,

$$
\begin{gathered}
\sigma^{2}\left[(G+H) \cos ^{4} \theta+(H+F) \sin ^{4} \theta\right. \\
\left.+2(N-H) \sin ^{2} \theta \cos ^{2} \theta\right] \\
+\sigma\left[K_{x} \cos ^{2} \theta+K_{y} \sin ^{2} \theta\right]=1 .
\end{gathered}
$$

Now, once the various parameters are determined using the relations shown in Equations 5 and 6 , and $(N-H)$ is determined as discussed below, Equation 10 is a quadratic equation which for a given value of $\theta$ will generally predict two roots of opposite sign and different magnitude (note the positive root is tensile and the negative is compressive). In this way, theoretical curves for tensile yield stress and compressive yield stress as functions of $\theta$ will result and can then be compared with experimental findings. Before doing this, there is one very crucial point that must be made clear. From Equation 5 it may be noted that,

$$
2 H=\frac{1}{C_{x} T_{x}}+\frac{1}{C_{y} T_{y}}-\frac{1}{C_{z} T_{z}}
$$

and

$$
2 N=\frac{1}{\tau_{x y}^{2}}(\text { see Hill [12]) }
$$

thus, one can conclude that $2(N-H)$, which itself is based upon a series of property values that are "constant" in a given sheet, must be a unique parameter. Since Equation 10 yields different values for $2(N-H)$ for variations in $\theta$ if the tensile and compressive yield stresses are not numerically equal, it may be concluded that only one condition will provide the unique value of $2(N-H)$. This is satisfied where,

*Exceptions have been noted; see e.g. [13-15].

$$
\sigma\left[K_{x} \cos ^{2} \theta+K_{y} \sin ^{2} \theta\right]=0 .
$$

A trivial solution occurs when $\sigma=0$, so we conclude that Equation 13 must be satisfied when,

$$
\tan ^{2} \theta=-\frac{K_{x}}{K_{y}} .
$$

This value of $\theta$ occurs where the absolute values of the tensile and compressive yield stresses are equal. In ending this discussion it may be noted that the above considerations do not enter if one uses the usual form of the Hill criterion since as may be seen from Equation 9, no linear terms involving $\sigma$ exist.

\section{Comparison of theory and experiment}

From reference [11], where a series of tensile and compressive stress-strain curves are given for various $\theta$ in a sheet of oriented polypropylene, we have selected pertinent information. As explained elsewhere [5], we would prefer to define a "yield stress" by the use of a consistent offset rather than sometimes using deviations from linearity and other times using the maxima in the load curves. Without accurate raw data, however, we have been forced to do the following.

1. Where a decided load maximum was obvious, the corresponding stress (note this is a nominal and not a true stress) was selected as the yield stress.

2. Where a distinct load maximum was not present, the back-extrapolation method (see e.g. Fig. $3 b$ of [10]) was used.

To avoid any confusion, Table I lists the values of tensile and compressive yield stress as a function of $\theta$ which we have chosen from [11].

TABLE I Tensile and compressive yield stress for angle $\theta$ from the work of Shinozaki and Groves [11]

\begin{tabular}{cll}
\hline $\begin{array}{l}\text { Angle to stretching Tensile yield } \\
\text { direction (degrees) }\end{array}$ & stress $\left(\mathrm{kg} \mathrm{mm}^{-2}\right)$ & $\begin{array}{l}\text { Compressive yield } \\
\text { stress }\left(\mathrm{kg} \mathrm{mm}^{-2}\right)\end{array}$ \\
\hline 0 & 24.5 & 4 \\
5 & 20.2 & 3.9 \\
10 & 16.0 & - \\
15 & - & 4.4 \\
20 & 10 & - \\
30 & - & 4.6 \\
45 & 4.5 & - \\
65 & 3.5 & 5.5 \\
85 & 2.9 & - \\
90 & 2.75 & 5.8 \\
\hline
\end{tabular}


From Equations 5 and 6, with $T_{x}=24.5$, $C_{x}=4, T_{y}=2.75$ and $C_{y}=5.8$ (all used as absolute values in $\mathrm{kg} \mathrm{mm}^{-2}$ ) we find:

$$
\begin{aligned}
& H+G=+0.0102 \\
& F+H=+0.0627 \\
& K_{x}=-0.209 \\
& K_{y}=+0.191
\end{aligned}
$$

From Equation 14,

$$
\tan ^{2} \theta=-\frac{-0.209}{0.191}=+1.095
$$

thus,

$$
\tan \theta=1.046 \operatorname{so} \theta=46^{\circ} 17^{\prime} \text {. }
$$

As this angle is so close to $45^{\circ}$ and as the accuracy of $K_{x}$ and $K_{y}$ is a little dubious because of difficulty in accurately establishing the various "yield stresses", we will for simplicity use the $45^{\circ}$ value to determine $2(N-H)$. From Table I, the equivalent value of tensile yield is 4.5 and it must, of course, be assumed that this same absolute value applies to the compressive yield which was not actually measured.

Now referring to Equation 10 using $\sigma=4.5$, $\theta=45^{\circ}$, and the values listed in Equation 15, one finds, $2(N-H)=+0.133$. Thus, from these data, Equation 10 may be rewritten as,

$\sigma^{2}\left[(0.0102) \cos ^{4} \theta+(0.0627) \sin ^{4} \theta\right.$

$\left.+(0.133) \sin ^{2} \theta \cos ^{2} \theta\right]$

$+\sigma\left[(0.191) \sin ^{2} \theta-(0.209) \cos ^{2} \theta\right]=1$.

By substituting various values for $\theta$ into Equation 16, each of which provides a positive (tensile) root and a negative (compressive) root, the predicted variations of these yield stresses as a function of $\theta$ result. The solid lines of Fig. 1 show these plots where absolute values are utilized. Actual experimental data from Table I are included and the agreement is seen to be excellent.

From the paper by Rawson and Rider [10], which involved oriented polyvinylchloride, their Fig. 5 must be consulted as individual stress strain curves were not included. Using the same approach as before, and estimating $T_{a}=10.4$, $C_{x}=8.4, T_{y}=8.4$, and $C_{y}=9.6$ we find,

$$
\begin{aligned}
H+G & =+0.0114 \\
F+H & =+0.0124 \\
K_{x} & =-0.0229 \\
K_{y} & =+0.0149
\end{aligned}
$$

Also, from Equation 14,

$$
\tan ^{2} \theta=-\frac{-.0229}{+.0149}=+1.535
$$

thus

$$
\tan \theta=1.24 \text { so } \theta=51^{\circ} 7^{\prime} .
$$

From the reference figure in $[10]$ it can be seen that the tensile and compressive yield stresses at an angle of $51^{\circ} 7^{\prime}$ are very close to equality; for our purposes a stress of 8.2 was chosen. The computed value of $2(N-H)$ is found to be +0.036 so the proper form of Equation 10 for these data is found to be,

$$
\begin{gathered}
\sigma^{2}\left[(0.0114) \cos ^{4} \theta-(0.0124) \sin ^{4} \theta\right. \\
\left.+(0.036) \sin ^{2} \theta \cos ^{2} \theta\right] \\
+\sigma\left[(0.0149) \sin ^{2} \theta-(0.0229) \cos ^{2} \theta\right]=1
\end{gathered}
$$

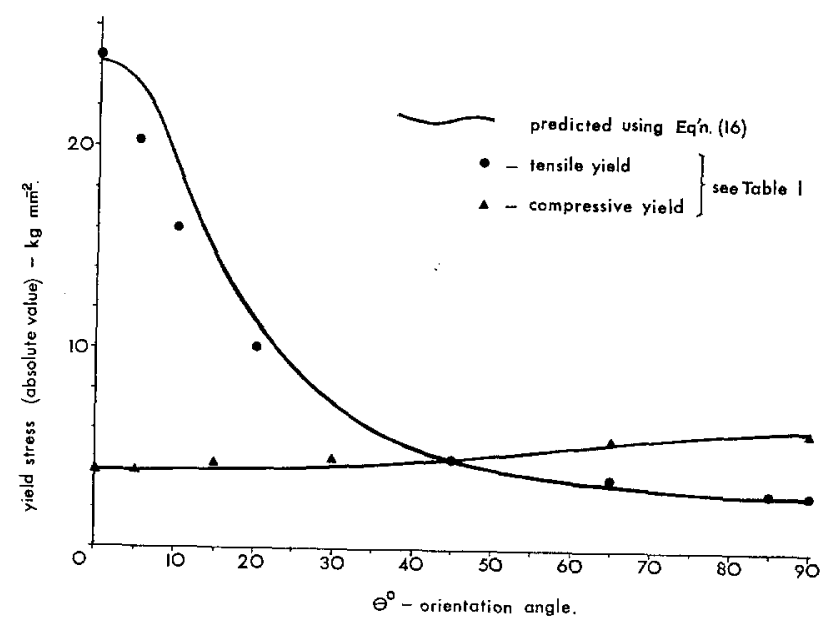

Figure 1 Tensile and compressive yield stress of oriented polypropylene as a function of orientation angle. Solid lines show theoretical predictions while the indicated points were determined experimentally by Shinozaki and Groves [11]. 


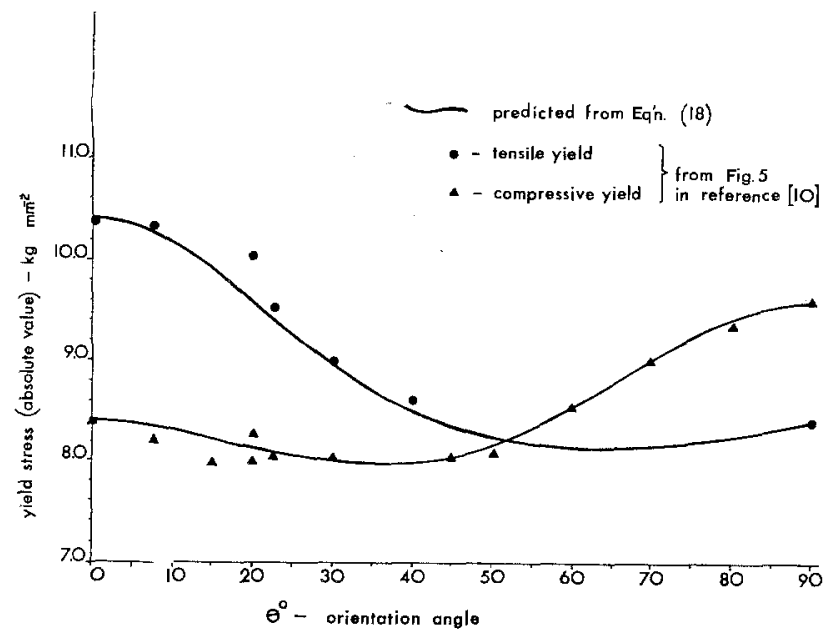

Figure 2 Tensile and compressive yield stress of oriented polyvinylchloride as a function of orientation angle. Solid lines show theoretical predictions while the indicated points were determined experimentally by Rawson and Rider [10].

Fig. 2 shows the predicted variations of yield stress as a function of $\theta$ with the actual experimental points from [10] superimposed. Again excellent agreement is found.

\section{Discussion}

Shinozaki and Groves [11] have suggested that a "three-part" criterion (owing to Kelley and Davies and used in composite work) might be appropriate for their findings in connection with tensile yield stress. However, they did not suggest how one could handle the prediction of compressive yield stress. We suggest that the excellent correlation seen in Fig. 1 supports our contention that the criterion expressed by Equation 4 as reduced to Equation 10 is quite adequate for the type of pressure-dependent solids being considered here.

Similar comments apply regarding the correlation seen in Fig. 2. Although in the paper by Rawson and Rider [10] they show a good correlation of experiment with prediction, we would point out that the equation they use for predictive purposes uses a value for $a_{x x}$ which seems incorrect according to their own definitions. No such parameter enters into our analysis.

\section{Conclusions}

A yield criterion for use with anisotropic polymeric solids has been shown to provide excellent correlation with published experimental data. Since it accounts for pressure dependency of yield behaviour, it seems to be more fundamentally sound than those proposed in earlier studies.

\section{References*}

1. W. Whit ney and R. D. Andrews, J. Polymer Sci. C, 16 (1967) 2981.

2. S. STERNSTEIN and L. ONGCHIN, A.C.S. Polymer Prep. 10 (1969) 1117.

3. P. B. BOWDEN and J. A. JUkES, J. Mater, Sci. 7 (1972) 52 .

4. J. C. BauWens, J. Polymer Sci. A-2, 8 (1970) 893.

5. R. S. RAGHAVA, R. M. CADDELL, and G. S.Y. YEH, J. Mater. Sci. 8 (1973) 225.

6. R. S. RAGHAVA, Ph.D. Dissertation, the University of Michigan (December 1972).

7. R.M.CADDELL, R.S. RAGHAVA, and A. G. ATKINS, Mater. Sci. and Eng. (1973).

8. N. BROWN, R. A. DUCKETT, and I. M. WARD, Phil. Mag. 18 (1968) 483.

9. J. Rider and E. HARGReaves, J. Polymer Sci. A-2, 7 (1969) 829.

10. F.F. RAWSON and J. G. RIDER, ibid $C, 33$ (1971) 87.

*After this paper had been accepted for publication, we learned that in 1969 in the Italian Journal Meccanica, 4, IV, p. 349, Professor F. Stassi-D'Alia had extended his yield criterion [see for example 5, 6] to include anisotropy. It is written in terms of principal stresses, and our Equation 4, rewritten to neglect all shear stresses, would be equivalent to Stassi-D'Alia's criterion. However, to analyse the type of yield information from sheet studies as we have done, the more generalized equation developed independently by the present authors must be used. 
11. D. Shinozaki and G. W. Groves, J. Mater. Sci. 14. A. N. Bramley and P. B. mellor, Int.J. Mech. Sci. 8 (1973) 71. $8(1966) 101$.

12. R. HILL, "Plasticity" (Clarendon Press, Oxford, 15. Idem, ibid 10 (1968) 211. 1950) pp. 317-20.

13. D. LEE and W. A. BACKofen, Trans. Met. Soc. Received 24 April and accepted 23 May 1973. AIME, 236 (1966) 1696. 